\title{
Upregulated forkhead-box A3 elevates the expression of forkhead-box A1 and forkhead-box A2 to promote metastasis in esophageal cancer
}

\author{
BING CHEN $^{1}$, JIEGEN YU ${ }^{2}$, LINMING LU ${ }^{1}$, FANGYUAN DONG ${ }^{1}$, FANGFANG ZHOU $^{1}$, \\ XIANGXIANG TAO ${ }^{1}$ and ENTAO $\mathrm{SUN}^{3}$ \\ ${ }^{1}$ Department of Pathology, School of Basic Medical Sciences; \\ ${ }^{2}$ Department of Management Science, School of Humanities and Management; \\ ${ }^{3}$ Department of Health Inspection and Quarantine, School of Laboratory Medicine, \\ Wannan Medical College, Wuhu, Anhui 241002, P.R. China
}

Received July 19, 2018; Accepted February 4, 2019

DOI: $10.3892 / \mathrm{ol} .2019 .10078$

\begin{abstract}
Esophageal cancer (EC) is one of the most lethal cancers currently known. Members of the forkhead-box A (FOXA) family, including FOXA1 and FOXA2, have been reported to regulate EC progression. However, the role of FOXA3, which is another FOXA member, has not yet been investigated. In the present study, public dataset analyses and immunohistochemistry of 96 samples from patients with EC were performed to determine the potential roles of FOXA3 in EC. The results revealed that FOXA3 was significantly upregulated in EC tumor tissues and Barrett's esophagus tissues. In addition, FOXA3 upregulation was positively associated with tumor invasion, distant metastasis, tumor-node-metastasis stage and shorter overall survival in patients with EC, and multivariate analysis identified FOXA3 as an independent prognostic marker. In vitro experiments demonstrated that the migratory and invasive abilities of EC109 and EC9706 cell lines were inhibited following FOXA3 knockdown. Notably, FOXA3 expression levels were positively correlated with FOXA1 and FOXA2 expression levels according to The Cancer Genome Atlas dataset analysis. Furthermore, FOXA3 knockdown decreased the expression levels of FOXA1 and FOXA2 in EC109 and EC9706 cell lines. Conversely, FOXA1
\end{abstract}

Correspondence to: Professor Entao Sun, Department of Health Inspection and Quarantine, School of Laboratory Medicine, Wannan Medical College, 22 Wenchang West Road, Wuhu, Anhui 241002, P.R. China

E-mail: 411134829@qq.com

Professor Bing Chen, Department of Pathology, Schools of Basic Medical Sciences, Wannan Medical College, 22 Wenchang West Road, Wuhu, Anhui 241002, P.R. China

E-mail: chenbing@wnmc.edu.cn

Key words: esophageal cancer, forkhead-box A3, metastasis, forkhead-box A2, compensation or FOXA2 overexpression compensated for the effects of FOXA3 knockdown on the migratory and invasive capacities of EC cells. In conclusion, the present study demonstrated that FOXA3 upregulation in EC cells promoted metastasis through regulation of other FOXA members.

\section{Introduction}

Esophageal cancer (EC) is the fifth most commonly diagnosed type of cancer and the fourth most common cause of cancer-associated mortality in China (1). Half of the newly diagnosed cases of esophageal squamous cell carcinoma (ESCC) every year occur in China. High incidence and mortality rates of ESCC have been reported in certain areas, including the Taizhou region in eastern China, the Taihang Mountains region in north-central China and Chaoshan district in Guangdong province (2,3). The highest risk factors for oral cancer and EC are tobacco smoke and high alcohol consumption, which jointly account for $\sim 90 \%$ of all cases (4). Most patients with EC are diagnosed in middle or advanced stages, and despite recent clinical advances in EC treatment, the 5-year survival rate is $<10 \%$ (5). Existing therapies include chemotherapy and radiotherapy, which display limited effectiveness, essentially due to the high incidence of metastasis (1). It is therefore crucial to determine the key factors that regulate EC metastasis in order to determine novel antitumor targets and extend the survival of patients with EC.

Forkhead-box (FOX) A (FOXA) functions as a transcription factor in numerous biological and pathological processes (6). The FOXA family includes three members, FOXA1, FOXA2 and FOXA3, which contain a highly conserved winged-helix forkhead DNA-binding domain that is involved in transcriptional regulation and DNA repair (7). All FOXA members contain overlapping patterns of expression in certain organs derived from the embryonic endoderm, including liver, stomach and intestine (6), and are involved in the establishment of developmental competence for these tissues $(8,9)$. Previous studies that used FOXA1- or FOXA2-specific knockout mice reported that FOXA1 and 
FOXA2 can compensate for the function of one another in a cell type-specific manner $(10,11)$. Additional studies demonstrated that FOXA1 and FOXA2 present overlapping roles in development and differentiation $(12,13)$. However, whether FOXA3 has similar compensatory roles for the function of other FOXA members remains unknown.

FOXA members can modify the transcription factors network, and modulate metaplasia and neoplasia of endoderm-derived tissues in adults (14-16). Previous studies have reported that FOXA1 is strongly associated with tumor progression, particularly metastasis in various types of cancer $(17,18)$. Furthermore, FOXA2 can promote tumor growth and metastasis $(19,20)$. In EC, genomic location analysis revealed that a restriction on FOXA transcriptional activation is progressively lost during progression of Barrett's esophagus toward adenocarcinoma (21). In addition, it has been reported that FOXA1 is overexpressed and associated with extensive lymph node metastasis in EC $(22,23)$, and that FOXA2 is upregulated in Barrett's metaplasia, dysplasia and adenocarcinoma (24). However, to the best of our knowledge, the expression and potential functions of FOXA3 in EC have not yet been identified.

The present study aimed to determine the potential roles of FOXA3 in EC progression. To do so, the expression levels of FOXA3 were evaluated in EC tumor tissues. Subsequently, the association between FOXA3 expression levels and clinical characteristics of patients with EC was determined. In vitro and in vivo experiments were also performed to evaluate the effects of FOXA3 on EC, and to highlight the potential FOXA3 mechanisms of action in the regulation of EC progression.

\section{Materials and methods}

Patients and specimens. Tumor specimens, including 96 pairs of EC tissues and adjacent nontumor tissues, were obtained from patients with EC who underwent surgical resection without preoperative treatment and without other tumors between April 2004 and May 2008 at the Department of General Surgery, The First Affiliated Hospital Wannan Medical College (Wuhu, China). All samples were checked manually by a pathologist to confirm the tumor type and purity. Histological analysis of tumors confirmed that patients had EC. Patients included in the study had SCC, undifferentiated carcinoma or Siewert type I esophagogastric junctional adenocarcinoma. The clinicopathological and baseline demographic characteristics of patients were retrospectively collected and comprised age, sex, tumor size, tumor site and tumor stage. Tumor stages were histologically classified according to the seventh edition of the American Joint Committee on Cancer tumor-node-metastasis (TNM) classification (25). Overall survival (OS) was calculated from the date of surgery to the date of death or the last follow-up. Follow-up was terminated in December 2011. The study was approved by the Research Medical Ethics Committee of Wannan Medical College (Wuhu, China) and was carried out in accordance with approved guidelines. All patients provided written informed consent for the use of clinical specimens. All fresh specimens were fixed with $4 \%$ formalin at $4^{\circ} \mathrm{C}$ overnight.
Immunohistochemistry (IHC). IHC was performed on formalin-fixed paraffin-embedded surgical specimens. Tissue sections were incubated at $60^{\circ} \mathrm{C}$ for $6 \mathrm{~h}$, deparaffinized in xylene, and rehydrated in a descending gradient of ethanol. Endogenous peroxidase activity was quenched with $3 \%$ hydrogen peroxide at room temperature for $10 \mathrm{~min}$. Following antigen retrieval through incubation with citrate buffer in a microwave oven at $95^{\circ} \mathrm{C}$ for $10 \mathrm{~min}$, sections were incubated with anti-FOXA3 antibody (1:300, cat. no. ab238112; Abcam, Cambridge, UK) at $4^{\circ} \mathrm{C}$ overnight. Tissue sections were then treated with Primary Antibody Amplifier Quanto for $10 \mathrm{~min}$ at room temperature and horseradish peroxidase (HRP) Polymer Quanto for $10 \mathrm{~min}$ at room temperature (Thermo Fisher Scientific, Inc., Waltham, MA, USA). Sections were visualized using a Nikon Eclipse Ti-s microscope (Nikon Corporation, Tokyo, Japan) after staining with 3'-diaminobenzidine for $2 \mathrm{~min}$ and counterstaining with hematoxylin for $5 \mathrm{~min}$ at room temperature. Immunostaining intensity was evaluated by two independent pathologists without knowledge of the clinicopathological data. The staining intensity was sorted as follows: 0 (negative), 1 (weak), 2 (moderate) and 3 (strong). According to the extent of staining, the staining area was scored as $0,1,2$, 3 or 4 when it contained $<5,5-25,26-50,51-75$ or $>75 \%$ positively stained cells, respectively. The IHC score was processed by multiplying the staining intensity with the staining area, which yielded a result ranging from 0 to 12 .

Cell lines. The human EC cell lines EC109 and EC9706 were obtained from the Shanghai Institutes for Biological Sciences (Shanghai, China). All cells were cultured in Dulbecco's modified Eagle's medium (DMEM; Sigma-Aldrich; Merck KGaA, Darmstadt, Germany) supplemented with $10 \%$ fetal bovine serum (FBS; Gibco; Thermo Fisher Scientific, Inc.) and cultured at $37^{\circ} \mathrm{C}$ in a humidified incubator containing $5 \% \mathrm{CO}_{2}$. To generate EC9706-luc cells that stably express luciferase, EC9706 cells at $80 \%$ confluence in a $10-\mathrm{cm}$ dish were transfected with $10 \mu \mathrm{g}$ pcDNA3.1/Luc $(0.01 \mu \mathrm{g} / \mu \mathrm{l}$; plasmid 32904; Addgene, Inc., Cambridge, MA, USA) in Lipofectamine ${ }^{\circledR}$ (Invitrogen; Thermo Fisher Scientific, Inc.) and serum-free medium at $37^{\circ} \mathrm{C}$ for $18-72 \mathrm{~h}$, and treated with $400 \mathrm{mg} / \mathrm{l} \mathrm{G} 418$ (Sigma-Aldrich; Merck KGaA) in 10\% FBS-supplemented DMEM. Culture media containing G418 was refreshed every week. After 3 weeks of G418 selection, isolated colonies were further purified by limiting dilution cloning, and single clones were maintained in DMEM with 200 mg/l G418.

Reverse transcription-quantitative polymerase chain reaction $(R T-q P C R)$. Total RNA was isolated from cells and tissue samples using TRIzol ${ }^{\circledR}$ reagent (Invitrogen; Thermo Fisher Scientific, Inc.). RNA was reverse transcribed using the PrimeScript RT reagent kit (cat. no. RR037; Takara Biotechnology Co., Ltd., Dalian, China) according to the manufacturer's protocol, and then processed for qPCR using SYBR Premix Ex Taq (cat. no. RR390; Takara Biotechnology Co., Ltd.) according to the manufacturer's protocol. Briefly, the $\mathrm{qPCR}$ reaction mixture was preheated to $95^{\circ} \mathrm{C}$ for $10 \mathrm{sec}$, followed by 35 cycles consisting of $95^{\circ} \mathrm{C}$ for $15 \mathrm{sec}, 65^{\circ} \mathrm{C}$ for $30 \mathrm{sec}$ and $72^{\circ} \mathrm{C}$ for $30 \mathrm{sec}$. GAPDH was used as an internal control. FOXA1, FOXA2, FOXA3 and GAPDH primers were designed as follows: FOXA1, forward 5'-GCAATA 
A

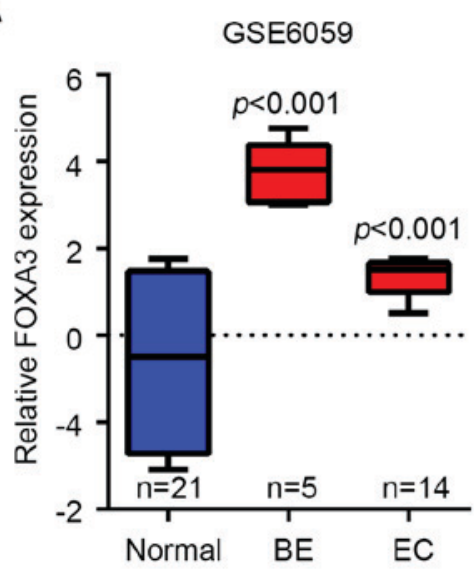

B

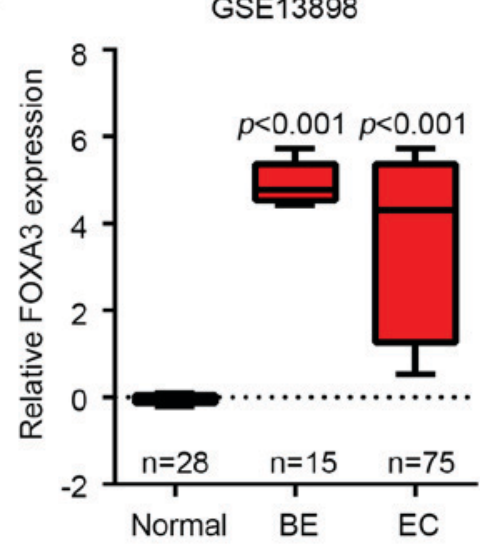

C

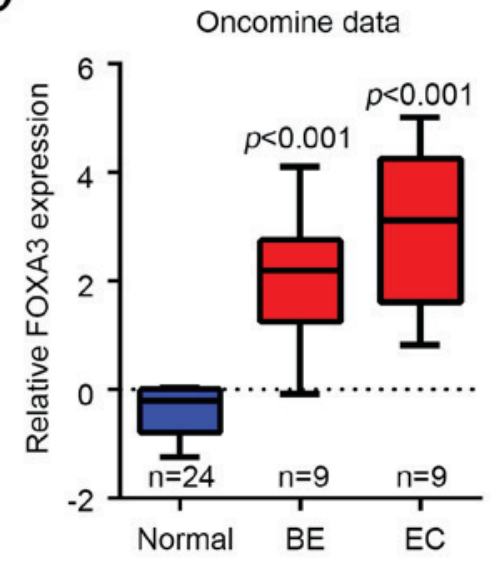

E

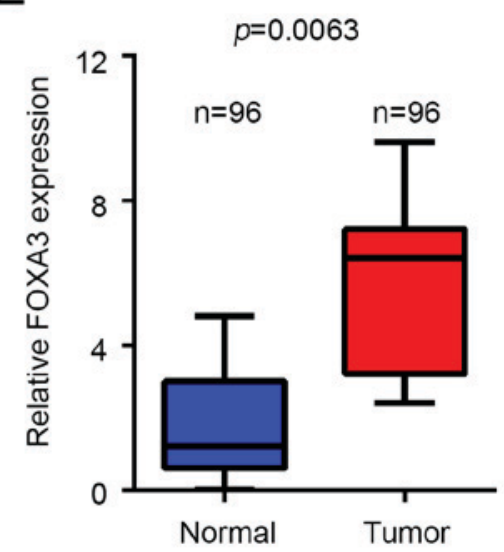

Figure 1. FOXA3 expression patterns in EC tissues. (A-C) Relative mRNA expression levels of FOXA3 in EC, BE and normal esophageal tissues from different public datasets. (D) Representative IHC staining images of FOXA3 and regional magnification in EC and normal tissues. Scale bar, $200 \mu$ m. (E) IHC score of FOXA3 expression in EC and normal tissues. BE, Barrett's esophagus; EC, esophageal cancer; FOXA3, forkhead-box A3; IHC, immunohistochemistry.

CTCGCCTTACGGCT-3', reverse 5'-TACACACCTTGGTAG TACGCC-3'; FOXA2, forward 5'-GGAGCAGCTACTATG CAGAGC-3', reverse 5'-CGTGTTCATGCCGTTCATCC-3'; FOXA3, forward 5'-GAGATGCCGAAGGGGTATCG-3', reverse 5'-TGATTCTCCCGGTAGTAAGGG-3'; and GAPDH, forward 5'-GAGTCAACGGATTTGGTCGT-3' and reverse 5'-TTGATTTTGGAGGGATCTCG-3'. The relative expression levels were calculated and normalized using the $2^{-\Delta \Delta \mathrm{Cq}}$ method (26).

Western blotting. Proteins were extracted from tissues or cells using Tissue or Cell Total Protein Extraction kit (Sangon Biotech Co., Ltd., Shanghai, China), and quantified using the Pierce Bicinchoninic Acid Protein Assay kit (Thermo Fisher Scientific, Inc.). Subsequently, $50 \mathrm{ng}$ total protein was separated by $10 \%$ SDS-PAGE and transferred onto polyvinylidene fluoride membranes. Membranes were blocked with $3 \%$ bovine serum albumin (Sigma-Aldrich; Merck KGaA) in PBS for $1 \mathrm{~h}$ at room temperature, and then incubated with primary antibodies against FOXA3 (1:1,000, cat. no. ab108454; Abcam), E-cadherin (1:1,000, cat. no. 20874; ProteinTech Group, Inc., Chicago, IL, USA), N-cadherin (1:1,000, cat. no. 22018; ProteinTech Group, Inc.), Snail (1:1,000, cat. no. ab53519; Abcam), Twist (1:1,000, cat. no. 25465; ProteinTech Group,
Inc.), FOXA1 (1:1,000, cat. no. 20411; ProteinTech Group, Inc.), FOXA2 (1:1,000, cat. no. 22474; ProteinTech Group, Inc.) and GAPDH (1:3,000, cat. no. 60004; ProteinTech Group, Inc.) at $4^{\circ} \mathrm{C}$ overnight. Subsequently, membranes were incubated with HRP-conjugated secondary antibodies $(1: 2,000$, cat. nos. 705-035-003, 115-035-003 and 111-035-003; Jackson ImmunoResearch Laboratories, Inc., West Grove, PA, USA) at room temperature for $2 \mathrm{~h}$. Enhanced chemiluminescence reagent (Pierce ${ }^{\mathrm{TM}}$ ECL Western Blotting Substrate; Thermo Fisher Scientific, Inc.) and a chemiluminescence imager (Tanon 4800; Tanon Science and Technology Co., Ltd., Shanghai, China) was used to detect the signal on the membrane. The intensity of the bands was analyzed using Quantity One Version 4.62 software (Bio-Rad Laboratories, Inc., Hercules, CA, USA).

Small interfering (si)RNA and overexpression. The targeting sequence for FOXA3 siRNA was 5'-GACGCGCCCTACAAC TTCAA-3'. The coding sequences of FOXA1 and FOXA2 were constructed into p3xFlag-CMV7 (Sigma-Aldrich; Merck KGaA). Transfection of siRNA and overexpression plasmids into EC cells was carried out using Lipofectamine ${ }^{\circledR} 2000$ (Invitrogen; Thermo Fisher Scientific, Inc.) according to the manufacturer's protocol. Briefly, cells were plated $24 \mathrm{~h}$ prior 


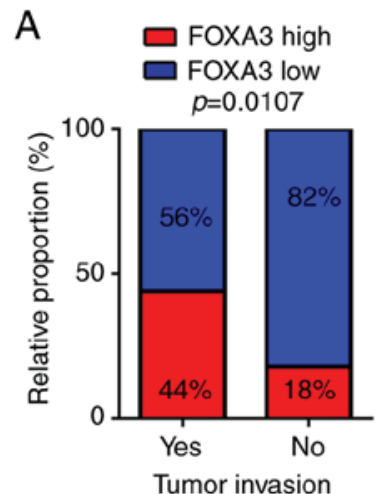

$\mathrm{D}$



$\mathrm{F}$

Multivariate cox regression for OS

Tumor invasion (yes vs no)

TNM stage (II-IV vs I)

FOXA3 expression (low vs high)





$\mathrm{E}$

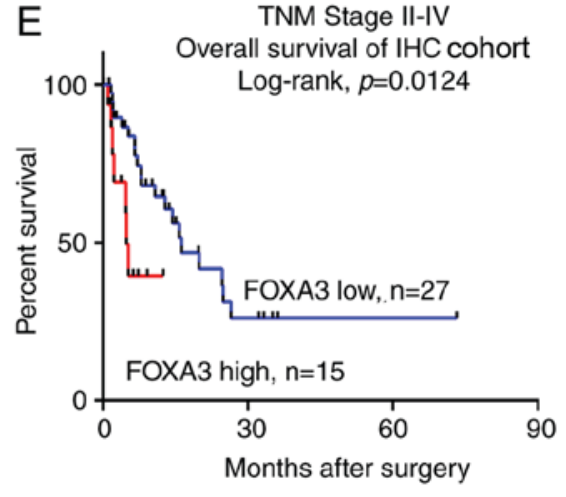

$\mathrm{HR}(95 \% \mathrm{Cl}) \quad P$-value

$1.062(0.494-2.338) \quad 0.394$

$1.337(0.525-2.887) \quad 0.044$

$3.873(1.233-8.995) \quad 0.045$

Figure 2. Predictive value of FOXA3 expression in patients with EC. (A and B) Association analysis of FOXA3 expression levels with various clinicopathological features. (C-E) Kaplan-Meier survival analysis demonstrated the association between FOXA3 expression and OS in patients at (C) TNM I-IV, (D) TNM I and (E) TNM II-IV. (F) Cox multivariate analysis for identification of the independent prognostic factors for OS of patients with EC. EC, esophageal cancer; FOXA1, forkhead-box A1; FOXA2, forkhead-box A2; FOXA3, forkhead-box A3; HR, hazard ratio; IHC, immunohistochemistry; OS, overall survival; TNM, tumor-node metastasis.

to transfection, and transfection was performed once cells reached $80 \%$ confluence. Overexpression plasmids $(10 \mu \mathrm{g}$ for cells in a $10-\mathrm{cm}$ dish, $0.01 \mu \mathrm{g} / \mu \mathrm{l})$ or siRNA (10 $\mu \mathrm{g}$ for cells in a 10 -cm dish, $0.01 \mu \mathrm{g} / \mu \mathrm{l})$ were mixed with Lipofectamine ${ }^{\circledR}$ and serum-free medium at room temperature for $20 \mathrm{~min}$. Subsequently, the mixture was added to the culture medium and incubated with the cells for 18-72 $\mathrm{h}$ under cell culture conditions. For the overexpression experiments, p3xFlag-CMV7 vectors were used as a control. For knockdown assays, scrambled control (5'-GCTCGACAGCCCTAACCTAA-3'; Invitrogen; Thermo Fisher Scientific, Inc.) was used.

Wound healing assay. Cells (1x106/ $/ \mathrm{ml})$ were seeded in 6-well plates and allowed to attach. Subsequently, a wound was generated with a $10-\mu 1$ pipette tip and cells were washed three times with PBS and incubated with $2 \mathrm{ml}$ complete medium. The migration status was observed under Nikon Eclipse Ti-s microscope (Nikon Corporation) at 0,12 and $24 \mathrm{~h}$ from the same point of view. The wound healing rate was measured with ImageJ version 1.42q software (National Institutes of Health, Bethesda, MD, USA).

Transwell assays. Transwell migration and invasion assays were performed in 12-well Transwell plates (pore size, $8 \mu \mathrm{m}$ ) according to the manufacturer's protocol (Corning Inc., Corning, NY, USA). For the invasion assay only, the Transwell lower chamber was coated with BD Matrigel ${ }^{\mathrm{TM}}$ Basement Membrane Matrix (BD Biosciences, San Jose, CA, USA). Cells $\left(1 \times 10^{5}\right)$ in serum-free medium were seeded into the Transwell upper chamber, whereas the lower chamber was filled with culture medium containing 20\% FBS, which served as a chemoattractant. The cell migratory and invasive capacities were evaluated at 24 and $48 \mathrm{~h}$, respectively. Uninvaded cells on the upper chamber were wiped off from the surface of the membrane by gentle rubbing, and invaded cells on the lower surface of the membrane were fixed with $4 \%$ paraformaldehyde 
Table I. Associations between FOXA3 expression levels and clinicopathological characteristics of patients with esophageal cancer.

\begin{tabular}{|c|c|c|c|c|}
\hline \multirow[b]{3}{*}{ Variables } & \multirow[b]{3}{*}{$\mathrm{N}$} & \multicolumn{2}{|c|}{$\begin{array}{c}\text { FOXA3 } \\
\text { expression }\end{array}$} & \multirow[b]{3}{*}{ P-value } \\
\hline & & High & Low & \\
\hline & & $\mathrm{N}(\%)$ & $\mathrm{N}(\%)$ & \\
\hline Age (years) & & & & 0.345779 \\
\hline$<60$ & 48 & $14(29)$ & $34(71)$ & \\
\hline$\geq 60$ & 48 & $10(21)$ & $38(79)$ & \\
\hline Sex & & & & 0.118824 \\
\hline Male & 57 & $11(19)$ & $46(81)$ & \\
\hline Female & 39 & $13(33)$ & $26(67)$ & \\
\hline Tumor size $(\mathrm{cm})$ & & & & 0.555604 \\
\hline$\leq 5$ & 47 & $13(28)$ & $34(72)$ & \\
\hline$>5$ & 49 & $11(22)$ & $38(78)$ & \\
\hline Nodal metastasis & & & & 0.155855 \\
\hline Yes & 52 & $16(31)$ & $36(69)$ & \\
\hline No & 44 & $8(18)$ & $36(82)$ & \\
\hline Tumor invasion & & & & 0.010738 \\
\hline Yes & 25 & $11(44)$ & $14(56)$ & \\
\hline No & 71 & $13(18)$ & $58(82)$ & \\
\hline Distant metastasis & & & & 0.063399 \\
\hline Yes & 5 & $3(60)$ & $2(40)$ & \\
\hline No & 91 & $21(23)$ & $70(77)$ & \\
\hline TNM stage & & & & 0.032509 \\
\hline I & 54 & $9(17)$ & $45(83)$ & \\
\hline II-IV & 42 & $15(36)$ & $27(64)$ & \\
\hline
\end{tabular}

FOXA3, forkhead-box A3; TNM, tumor node metastasis.

for $10 \mathrm{~min}$ and stained with $0.1 \%$ crystal violet for $5 \mathrm{~min}$ at room temperature. The number of invasive and migratory cells was counted under Nikon Eclipse Ti-s microscope (Nikon Corporation) in five randomly selected fields.

Animal study. Male Balb/c nude mice (age, 4-6 weeks; weight, 15-20 g; housing conditions: $26^{\circ} \mathrm{C}, 50 \%$ humidity, 10/14-h light/dark cycle, free access to sufficient sterile food and water) were purchased from the Shanghai Laboratory Animal Center of the Chinese Academy Sciences (Shanghai, China) and were housed in a separate pathogen-free room. Animal care and experiments were approved by the Research Medical Ethics Committee of Wannan Medical College, and were performed in strict accordance with the approved guidelines. All mice were randomized in a controlled fashion (n=6/group). For the lung metastasis model, transfected EC9706-Luc cells were suspended in precooled PBS $\left(1 \times 10^{6}\right.$ cells/mouse in $100 \mu \mathrm{l}$ PBS $)$ and were injected through the tail vein. All mice were monitored for bioluminescence every week with IVIS200 imaging (Xenogen; Caliper Life Sciences, Hopkinton, MA, USA) following intraperitoneal administration of $200 \mu 1$ luciferin
(15 mg/ml; Promega Corporation, Madison, WI, USA). The luciferase signal intensity was calculated by region of interest analysis.

Statistical analysis. Patients were grouped according to the results of the receiver operating characteristic (ROC) analyses obtained from IHC data. An IHC score of 6 was determined as the ideal cutoff to divide patients into the low FOXA3 expression group (score, $0-6 ; n=70$ ) and high FOXA3 expression group (score, 7-12; $n=20$ ). The association between FOXA3 expression and clinicopathological characteristics of patients with EC was evaluated using $\chi^{2}$ test. Pearson correlation coefficients were calculated to analyze the correlation between FOXA3 and FOXA1 or FOXA2 expression levels in The Cancer Genome Atlas (TCGA) Esophageal Carcinoma datasets (https://portal.gdc.cancer.gov/projects/TCGA-ESCA) (27). Survival curves were constructed with the Kaplan-Meier method and compared using the log-rank test. Univariate and multivariate analyses were performed using Cox regression; the statistically significant characteristics in the univariate analysis were used to perform multivariate analysis. For comparisons between tumor and tumor-adjacent tissues, data from GSE6059 (https://www. ncbi.nlm.nih.gov/geo/query/acc.cgi?acc=GSE6059) (28), GSE13898 (https://www.ncbi.nlm.nih.gov/geo/query/acc. cgi?acc=GSE13898) (29) and Oncomine (https://www.oncomine.org) (30) datasets, and the IHC results were used. For comparisons between the control and siRNA-transfected groups, an unpaired Student's t-test was used. To compare FOXA3 expression in tumor and adjacent nontumor tissues following IHC analysis, a paired Student's t-test was used. For comparisons among multiple groups, One-way analysis of variance followed by Scheffe post hoc test was used. All statistical tests were two-tailed and $\mathrm{P}<0.05$ was considered to indicate a statistically significant difference. Data were analyzed using SPSS version 22.0 software (IBM Corp., Armonk, NY USA) and the R software version 3.2.2 (R Foundation for Statistical Computing, Vienna, Austria).

\section{Results}

FOXA3 is upregulated in EC. To determine whether FOXA3 was involved in EC progression, FOXA3 expression levels were analyzed in EC and adjacent normal tissues obtained from various public datasets. The results demonstrated that FOXA3 was significantly upregulated in EC tissues from GSE6059 [fold change $(\mathrm{FC})=4.234, \mathrm{P}<0.001$ ] (28), GSE13898 ( $\mathrm{FC}=12.373, \mathrm{P}<0.001)(29)$ and 'oncomine' $(\mathrm{FC}=8.368$, $\mathrm{P}<0.001)(30)$ datasets compared with in adjacent healthy tissues (Fig. 1A-C). The results also reported that FOXA3 expression was significantly upregulated in metaplasia tissues from Barrett's esophagus cases; $\mathrm{FC}=23.863, \mathrm{P}<0.001$; $\mathrm{FC}=32.219, \mathrm{P}<0.001$; and $\mathrm{FC}=4.490, \mathrm{P}<0.001$ for $\mathrm{GSE6059}$, GSE13898 and 'oncomine' datasets, respectively, compared with in adjacent healthy tissues (Fig. 1A-C). These data suggested that FOXA3 may be involved in EC progression and in tumorigenesis. FOXA3 protein levels were also determined in 96 pairs of EC and adjacent nontumor tissues by IHC. The results demonstrated that, although FOXA3 was present in both types of tissue, FOXA3 protein expression was upregulated in 
Table II. Univariate and multivariate Cox analyses of FOXA3 and clinicopathological characteristics of patients with esophageal cancer.

\begin{tabular}{|c|c|c|c|c|}
\hline \multirow[b]{2}{*}{ Variables } & \multicolumn{2}{|c|}{ Univariate analysis } & \multicolumn{2}{|c|}{ Multivariate analysis } \\
\hline & HR $(95 \%$ CI $)$ & P-value & HR $(95 \%$ CI) & P-value \\
\hline \multicolumn{5}{|l|}{ Age (years) } \\
\hline$\leq 60$ vs. $>60$ & $0.68(0.30-1.37)$ & 0.085 & & NS \\
\hline \multicolumn{5}{|l|}{ Sex } \\
\hline Male vs. female & $1.44(0.49-5.36)$ & 0.144 & & NS \\
\hline \multicolumn{5}{|l|}{ Tumor size (cm) } \\
\hline$\leq 5$ vs. $>5$ & $2.67(1.25-5.03)$ & 0.077 & & NS \\
\hline \multicolumn{5}{|l|}{ Nodal metastasis } \\
\hline Yes vs. no & $2.56(0.69-5.21)$ & 0.055 & & NS \\
\hline \multicolumn{5}{|l|}{ Tumor invasion } \\
\hline Yes vs. no & $1.06(0.49-2.34)$ & $0.011^{\mathrm{a}}$ & & NS \\
\hline \multicolumn{5}{|l|}{ Distant metastasis } \\
\hline Yes vs. no & $1.97(0.89-5.64)$ & $0.003^{\mathrm{a}}$ & & NS \\
\hline \multicolumn{5}{|l|}{ TNM stage } \\
\hline I vs. II-IV & $1.34(0.12-0.88)$ & $0.024^{\mathrm{a}}$ & & $0.044^{\mathrm{a}}$ \\
\hline FOXA3 expression & $3.87(1.23-8.99)$ & $0.035^{\mathrm{a}}$ & $3.11(1.57-7.66)$ & \\
\hline Low vs. high & $3.87(1.23-8.99)$ & & & $0.045^{\mathrm{a}}$ \\
\hline
\end{tabular}

${ }^{\text {a }} \mathrm{P}<0.05$. CI, confidence interval; HR, hazard ratio; FOXA3, forkhead-box A3; NS, not significant; TNM, tumor-node metastasis.

EC samples compared with in nontumor tissues $(\mathrm{P}=0.0126$; Fig. 1D and E).

FOXA3 upregulation is associated with tumor invasion and can be used as an independent prognostic marker in patients with EC. To evaluate the potential roles of FOXA3 in EC, the association between FOXA3 expression levels obtained from IHC assay and clinicopathological characteristics of patients with EC were calculated. The results revealed that FOXA3 upregulation was significantly associated with tumor invasion $(\mathrm{P}=0.0107)$ and advanced $\mathrm{TNM}$ stage $(\mathrm{P}=0.0325$; Fig. 2A and B; Table I). In addition, patients in the high FOXA3 expression group presented a poorer OS compared with patients in the low FOXA 3 expression group $(\mathrm{P}=0.0166$; Fig. 2C).

To further evaluate the efficiency of FOXA3 expression at distinguishing patients according to their TNM stages, patients were divided into early (stage I) and advanced (stages II-IV) groups. The results demonstrated that in both groups, FOXA3 levels could significantly predict patient outcomes (Fig. 2D and E). These data suggested that FOXA3 expression may be associated with the OS of patients with EC.

Univariate Cox analysis was used to identify the prognostic value of clinicopathological characteristics for OS in patients with EC. The results demonstrated that tumor invasion, distant metastasis, TNM stage and FOXA3 expression $(\mathrm{P}=0.011$, $0.003,0.024$ and 0.035 , respectively; Table II) represented risk factors that were positively associated with patient OS. Further adjustment of covariate factors using multivariate Cox analysis identified TNM stage $(\mathrm{P}=0.044)$ and upregulated
FOXA3 $(\mathrm{P}=0.045)$ as additional independent risk factors for EC (Fig. 2F). These data suggested that FOXA3 upregulation may be an independent factor that could predict poor prognosis in patients with EC.

FOXA3 promotes migration and invasion of EC cells. Since FOXA3 upregulation was significantly associated with tumor invasion in EC tissue (Fig. 2A), it was hypothesized that FOXA3 may be able to regulate metastasis in EC. To verify this hypothesis in vitro, EC109 and EC9706 cell lines were knocked-down for FOXA3, prior to measuring their migratory and invasive abilities. Knockdown efficiency was determined by RT-qPCR and western blotting (Fig. 3A and B). The results demonstrated that the migratory ability of EC cells following FOXA3 knockdown was significantly reduced according to the wound healing and Transwell migration assays $(\mathrm{P}<0.0001$; Fig. 3C and $\mathrm{D}$, respectively). Transwell invasion data further demonstrated that FOXA3 knockdown significantly inhibited the invasive ability of EC109 and EC9706 cell lines ( $\mathrm{P}<0.0001$; Fig. 3E).

$\mathrm{N}$-cadherin and E-cadherin protein expression levels were decreased and increased, respectively, in EC9706 cells following FOXA3 knockdown (Fig. 3F). In addition, $\mathrm{N}$-cadherin and E-cadherin mRNA expression levels were significantly decreased and increased, respectively, in EC9706 cells following FOXA3 knockdown (Fig. 3F). These results suggested that FOXA3 may regulate the epithelial-mesenchymal transition (EMT) in EC cells. However, although the protein levels of Snail, snail family transcriptional repressor 2 and twist family bHLH transcription factor were decreased following FOXA3 knockdown, no decrease in 
A

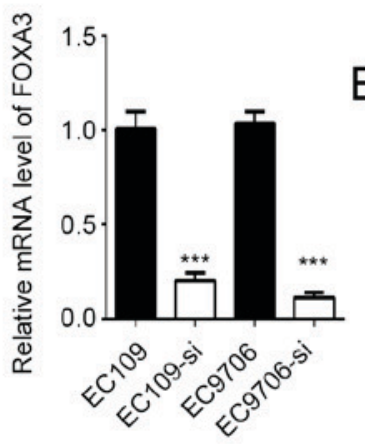

D

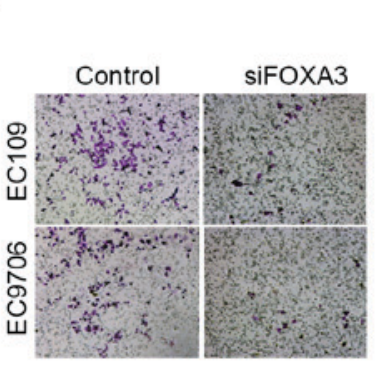

F

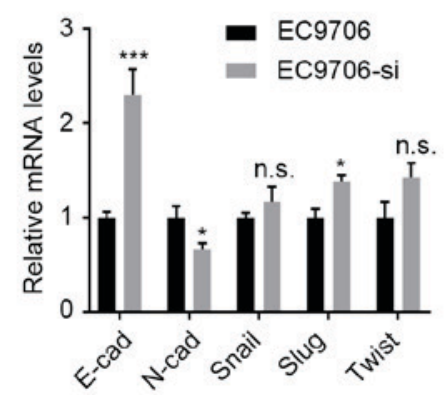

B

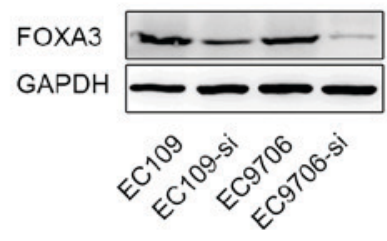

C
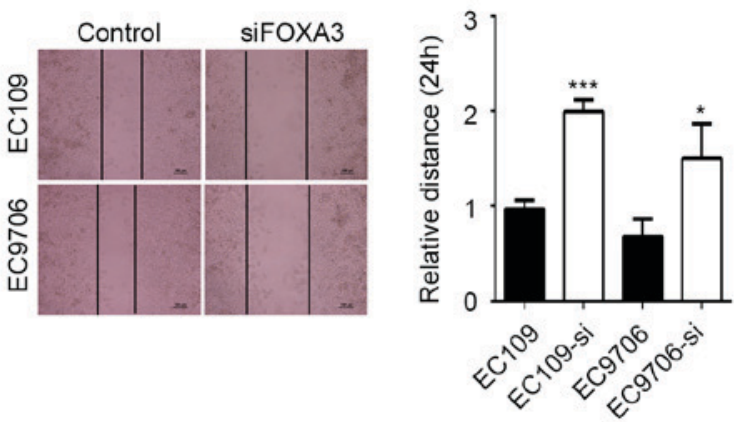

E
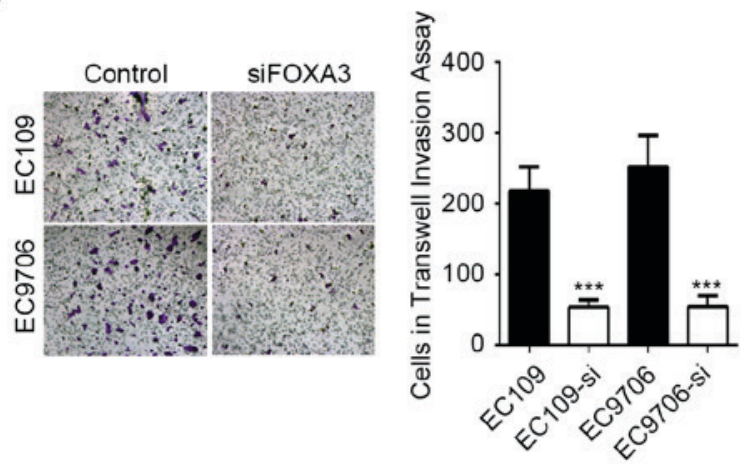

G

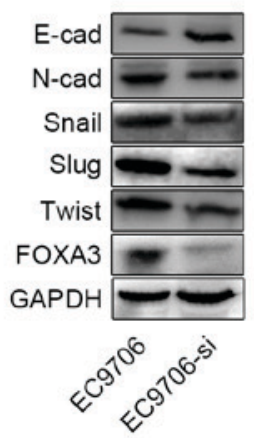

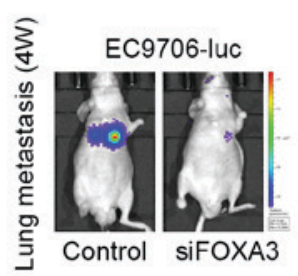

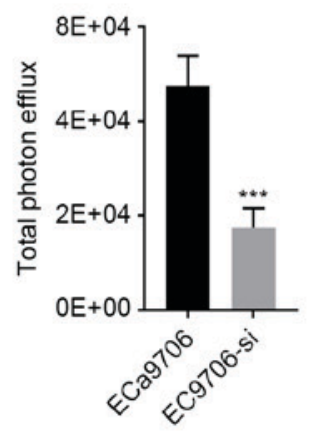

Figure 3. FOXA3 promotes migration and invasion of EC cells. FOXA3 knockdown efficiency in EC109 and EC9706 cells determined by (A) RT-qPCR and (B) western blotting. (C) Migratory ability of EC cells following FOXA3 knockdown determined by wound healing assay. Representative images (10x magnification) and statistical data are presented. (D and E) Migratory and invasive abilities of EC cells following FOXA3 knockdown determined by Transwell assay. Representative (20x magnification) and statistical data are presented. (F) mRNA (left) and protein (right) expression levels of the EMT-associated markers E-cad, N-cad, Snail, Slug, and Twist in EC9706 cells following FOXA3 knockdown. (G) Metastatic ability of EC cells following FOXA3 knockdown in vivo. Representative and statistical data are presented. All experiments were repeated at least three times. $\mathrm{P}<0.05$, ${ }^{* * *} \mathrm{P}<0.001$. E-cad, E-cadherin; FOXA3, forkhead-box A3; N-cad, N-cadherin; NS, not significant; si, small interfering; Slug, snail family transcriptional repressor 2; Twist, twist family bHLH transcription factor.

their mRNA expression levels was observed (Fig. 3F), which suggested that FOXA3 may regulate EMT through other processes.

The effects of FOXA3 on metastasis were also evaluated in vivo using EC9706-luc cells. The results demonstrated that FOXA3 knockdown significantly reduced lung metastasis originating from EC cells (Fig. 3G). Taken together, these data suggested that FOXA3 may regulate the metastatic potential of EC cells.

FOXA3 regulates the expression of other FOXA proteins. FOXA1 and FOXA2 are capable of regulating EC metastasis (17-20), and previous studies have reported that they can functionally compensate for each other (10-13). The present study therefore evaluated whether FOXA3 could compensate for FOXA1 and FOXA2 functions. The results demonstrated that the mRNA and protein expression levels of FOXA1 and FOXA2 were decreased in EC109 and EC9706 cells following FOXA3 knockdown (Fig. 4A and B). In addition, correlation analysis involving TCGA datasets revealed that FOXA3 expressions was positively correlated with FOXA1 $(\mathrm{R}=0.2141$, $\mathrm{P}=0.0034)$ and FOXA2 $(\mathrm{R}=0.6139, \mathrm{P}<0.0001)$ expression levels in EC tumor tissues (Fig. 4C). These data demonstrated that various FOXA members presented similar expression 
A
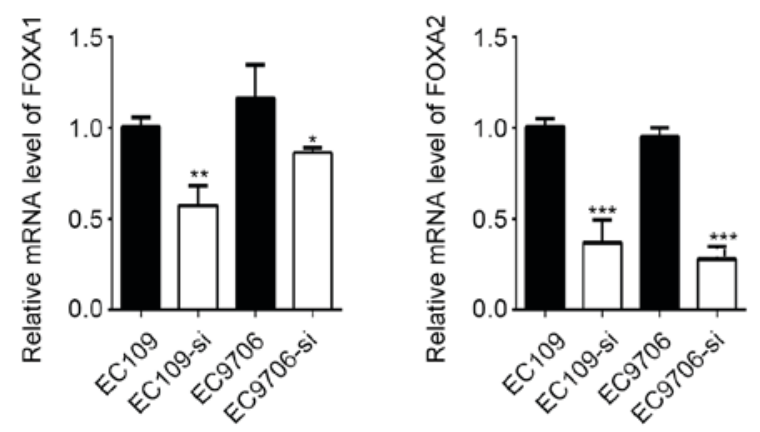

B

C

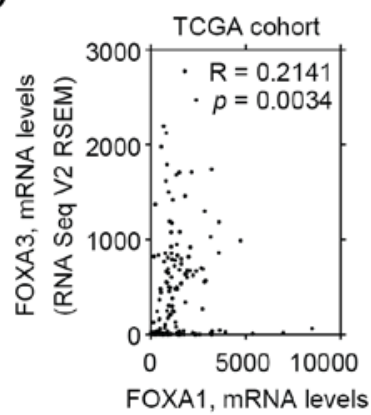

D
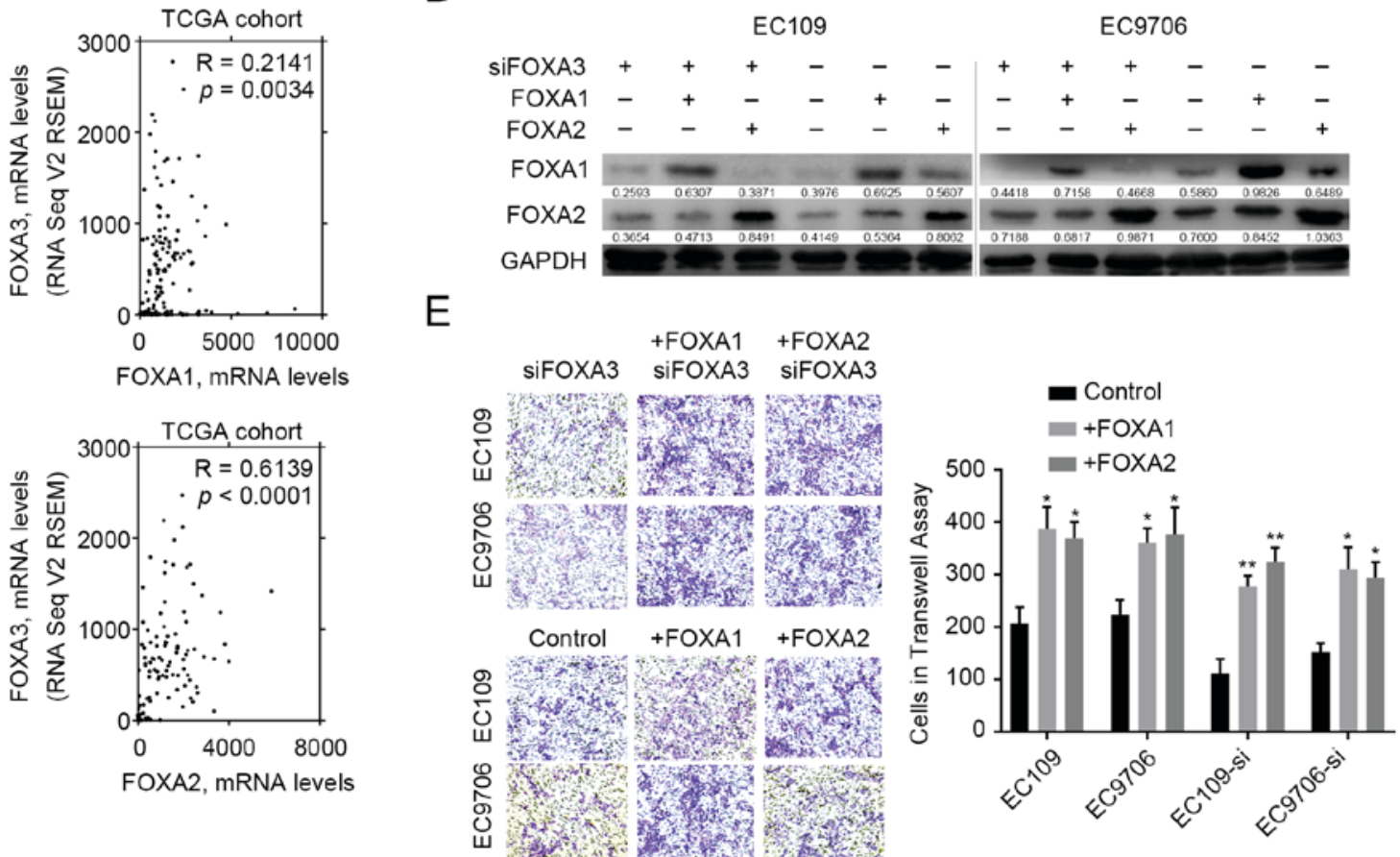

Figure 4. FOXA3 elevates the expression levels of other FOXAs in EC. (A) FOXA1 and FOXA2 mRNA expression levels in EC109 and EC9706 cells following FOXA3 knockdown determined by RT-qPCR. (B) FOXA1 and FOXA2 protein expression in EC109 and EC9706 cells following FOXA3 knockdown determined by western blotting. (C) Correlation analysis of FOXA1, FOXA2 and FOXA3 expression levels from in TCGA dataset. (D) FOXA2 overexpression efficiency on EC cells with FOXA3 knockdown determined by western blotting. Relative expression ratios of FOXA1/GAPDH or FOXA2/GAPDH are shown. (E) Effect of FOXA2 overexpression in EC cells with FOXA3 knockdown determined by Transwell invasion assays. Representative (20x magnification) and statistical data are presented. All experiments were repeated at least three times. ${ }^{*} \mathrm{P}<0.05 ;{ }^{* * *} \mathrm{P}<0.01 ;{ }^{* * * *} \mathrm{P}<0.001$. FOXA1, forkhead-box A1; FOXA2, forkhead-box A2; FOXA3, forkhead-box A3; si, small interfering; TCGA, The Cancer Genome Atlas.

patterns in EC tissues, which suggested that FOXA3 may be capable of regulating their expression. Subsequently, the present study induced overexpression of FOXA2 or FOXA1 in FOXA3-knocked-down EC cells (Fig. 4D). The results demonstrated that FOXA2 overexpression was capable of compensating for FOXA 3 loss of function and promoted tumor invasion (Fig. 4E). FOXA1 overexpression also compensated for the effects of FOXA3 knockdown (Fig. 4E).

\section{Discussion}

Clinicopathological characteristics, including tumor stage, nodal status and resection margin are generally used to determine prognosis and survival of patients with cancer (31). These characteristics can be determined according to the TNM classification system. However, due to large tumor heterogeneity, the efficiency of the TNM system is limited in EC (32). Novel diagnostic and prognostic tools are therefore required to improve patient survival and clinical treatment efficacy. The results from the present study suggested that FOXA3 may be used as an independent prognostic marker and as a potential invasiveness marker in patients with EC.

Similar to other FOXA members, FOXA3, which is also known as hepatocyte nuclear factor $3 \gamma$, serves certain roles in the development of endoderm tissues, particularly the liver and pancreas (33). The roles of FOXA1 and FOXA2 in cancer have been extensively investigated; however, to the best of our knowledge, only one study has reported that FOXA3 mRNA expression is increased in mouse liver tumors (34). Despite the lack of research on FOXA3 expression in human cancer, some 
studies have reported that abnormal expression of FOXA3 is observed in the metaplasia of various types of tissue $(35,36)$. For example, FOXA3 is upregulated in airway goblet cells of patients with asthma and chronic obstructive pulmonary disease (37). In addition, FOXA3 expression is significantly correlated with chloride channel accessory 1 , which is associated with smoke-induced mucin synthesis and further mucous metaplasia (38). These studies suggested that FOXA3 might be involved in cancer development. Furthermore, it has been reported that increased FOXA3 expression modulates age-associated metabolic disorder (39), which suggested that FOXA3 might serve a role in tumorigenesis, since senescence and cancer are closely associated. The results from the present study reported that FOXA3 was upregulated in Barrett's esophagus compared with in normal tissues, which further suggested that FOXA3 may serve certain roles in EC initiation. To the best of our knowledge, the present study was the first to quantify FOXA3 upregulation in EC tissues, and to demonstrate that FOXA3 was positively associated with metastasis and OS of patients with EC. These cancer-promoting effects of FOXA3 on EC progression may be similar to those of other FOXA members, although their functions present certain differences. For example, FOXA1 promotes lymph node metastasis (23), whereas FOXA3 has no significant effect on nodal metastasis. Notably, during definitive endoderm formation, FOXA3 is activated after other FOXA members, and might work differently than FOXA1 and FOXA2 (38-40). Furthermore, although the present study suggested that upregulated FOXA2 may compensate for decreased FOXA3 functions, it has also been reported that FOXA3 competes with FOXA2 for DNA binding in metaplasia and thus confers distinct functions by reducing FOXA2 activity (36). These studies, combined with results from the present study, suggested that, although similar expression levels of FOXA members were observed in EC tissues, switching FOXA3 and FOXA2 activation may be critical for regulating the metastatic inclination of EC cells toward lymphatic or hematogenous vessels. Furthermore, distinguishing the roles of FOXA2 and FOXA3 in EC progression may aid in the development of a more precise definition of invasive EC and treatment strategy for this pathology. The roles of FOXA3 and FOXA2 in EC remain poorly understand and require further investigation.

In conclusion, the results from the present study suggested that FOXA3 upregulation in EC may be considered as an independent prognostic factor, and that FOXA3 may regulate the migratory and invasive abilities of EC cells. In addition, FOXA3 modulated expression of other FOXA members, and FOXA2 upregulation compensated for the functions of FOXA3 in tumor invasion. Future studies will focus on the mechanisms underlying the roles of FOXA3 and its potential applications in the treatment of EC.

\section{Acknowledgements}

Not applicable.

\section{Funding}

The present study was supported by the National Natural Science Foundation of China (grant no. 31870352), the Foundation for Key Project of Natural Science Research Education
Department of Anhui Province (grant no. KJ2017A249) and the major Project of Education Department in Anhui (grant nos. KJ2016A725 and SK2018A0190).

\section{Availability of data and materials}

The datasets used and/or analyzed during the current study are available from the corresponding author on reasonable request.

\section{Authors' contributions}

BC performed all of the experiments. BC, JY and LL performed tissue analysis. BC and $\mathrm{FZ}$ carried out data analysis and interpretation; BC and ES carried out statistical analysis. FD and XT collected clinical data. BC wrote the manuscript, conceived the idea and designed the experiments. All authors read and approved the final manuscript.

\section{Ethics approval and consent to participate}

The study and animal experiments were approved by the Research Medical Ethics Committee of Wannan Medical College and were carried out in accordance with the ethical guidelines of the Declaration of Helsinki. All patients provided written informed consent.

\section{Patient consent for publication}

All patients provided written informed consent.

\section{Competing interests}

The authors declare that they have no competing interests.

\section{References}

1. Chen W, He Y, Zheng R, Zhang S, Zeng H, Zou X and He J: Esophageal cancer incidence and mortality in China, 2009. J Thorac Dis 5: 19-26, 2013.

2. Cook MB, Chow WH and Devesa SS: Oesophageal cancer incidence in the United States by race, sex, and histologic type, 1977-2005. Br J Cancer 101: 855-859, 2009.

3. Ferlay J, Shin HR, Bray F, Forman D, Mathers C and Parkin DM: Estimates of worldwide burden of cancer in 2008: GLOBOCAN 2008. Int J Cancer 127: 2893-2917, 2010.

4. Xu XC: Risk factors and gene expression in esophageal cancer. Methods Mol Biol 471: 335-360, 2009

5. Paulson TG and Reid BJ: Focus on Barrett's esophagus and esophageal adenocarcinoma. Cancer Cell 6: 11-16, 2004.

6. Besnard V, Wert SE, Hull WM and Whitsett JA: Immunohistochemical localization of Foxa1 and Foxa2 in mouse embryos and adult tissues. Gene Expr Patterns 5: 193-208, 2004.

7. Kaestner KH, Hiemisch H, Luckow B and Schütz G: The HNF-3 gene family of transcription factors in mice: Gene structure, cDNA sequence, and mRNA distribution. Genomics 20: 377-385, 1994.

8. Kaestner KH: The making of the liver: Developmental competence in foregut endoderm and induction of the hepatogenic program. Cell Cycle 4: 1146-1148, 2005.

9. Zaret K: Developmental competence of the gut endoderm: Genetic potentiation by GATA and HNF3/fork head proteins. Dev Biol 209: 1-10, 1999.

10. Augello MA, Hickey TE and Knudsen KE: FOXA1: Master of steroid receptor function in cancer. EMBO J 30: 3885-3894, 2011.

11. Gao N, Le Lay J, Qin W, Doliba N, Schug J, Fox AJ, Smirnova O, Matschinsky FM and Kaestner KH: Foxa1 and Foxa2 maintain the metabolic and secretory features of the mature beta-cell. Mol Endocrinol 24: 1594-1604, 2010. 
12. Ferri AL, Lin W, Mavromatakis YE, Wang JC, Sasaki H, Whitsett JA and Ang SL: Foxa1 and Foxa2 regulate multiple phases of midbrain dopaminergic neuron development in a dosage-dependent manner. Development 134: 2761-2769, 2007.

13. Mavromatakis YE, Lin W, Metzakopian E, Ferri AL, Yan CH, Sasaki H, Whisett J and Ang SL: Foxa1 and Foxa2 positively and negatively regulate Shh signalling to specify ventral midbrain progenitor identity. Mech Dev 128: 90-103, 2011.

14. Carroll JS, Liu XS, Brodsky AS, Li W, Meyer CA, Szary AJ, Eeckhoute J, Shao W, Hestermann EV, Geistlinger TR, et al: Chromosome-wide mapping of estrogen receptor binding reveals long-range regulation requiring the forkhead protein FoxA1. Cell 122: 33-43, 2005.

15. Gao N, White P and Kaestner KH: Establishment of intestinal identity and epithelial-mesenchymal signaling by Cdx2. Dev Cell 16: 588-599, 2009.

16. Slack JM: Metaplasia and transdifferentiation: From pure biology to the clinic. Nat Rev Mol Cell Biol 8: 369-378, 2007.

17. Robbins CM, Tembe WA, Baker A, Sinari S, Moses TY, Beckstrom-Sternberg S, Beckstrom-Sternberg J, Barrett M, Long J, Chinnaiyan A, et al: Copy number and targeted mutational analysis reveals novel somatic events in metastatic prostate tumors. Genome Res 21: 47-55, 2011.

18. Jain RK, Mehta RJ, Nakshatri H, Idrees MT and Badve SS High-level expression of forkhead-box protein A1 in metastatic prostate cancer. Histopathology 58: 766-772, 2011.

19. Salem M, O'Brien JA, Bernaudo S, Shawer H, Ye G, Brkić J, Amleh A, Vanderhyden BC, Refky B, Yang BB, et al: miRNA-590-3p promotes ovarian cancer growth and metastasis via a novel FOXA2-versican pathway. Cancer Res 78: 4175-4190, 2018.

20. Wang B, Liu G, Ding L, Zhao J and Lu Y: FOXA2 promotes the proliferation, migration and invasion, and epithelial mesenchymal transition in colon cancer. Exp Ther Med 16: 133-140, 2018.

21. Watts JA, Zhang C, Klein-Szanto AJ, Kormish JD, Fu J, Zhang MQ and Zaret KS: Study of FoxA pioneer factor at silent genes reveals Rfx-repressed enhancer at $\mathrm{Cdx} 2$ and a potential indicator of esophageal adenocarcinoma development. PLoS Genet 7: e1002277, 2011.

22. Lin L, Miller CT, Contreras JI, Prescott MS, Dagenais SL, Wu R, Yee J, Orringer MB, Misek DE, Hanash SM, et al: The hepatocyte nuclear factor 3 alpha gene, HNF3alpha (FOXA1) on chromosome band 14q13 is amplified and overexpressed in esophageal and lung adenocarcinomas. Cancer Res 62: 5273-5279, 2002.

23. Sano M, Aoyagi K, Takahashi H, Kawamura T, Mabuchi T, Igaki $\mathrm{H}$, Tachimori Y, Kato H, Ochiai A, Honda H, et al: Forkhead box A1 transcriptional pathway in KRT7-expressing esophageal squamous cell carcinomas with extensive lymph node metastasis. Int J Oncol 36: 321-330, 2010.

24. Wang DH, Tiwari A, Kim ME, Clemons NJ, Regmi NL, Hodges WA, Berman DM, Montgomery EA, Watkins DN, Zhang X, et al: Hedgehog signaling regulates FOXA2 in esophageal embryogenesis and Barrett's metaplasia. J Clin Invest 124 3767-3780, 2014

25. Edge SB, Byrd DR, Compton CC, et al: AJCC Cancer Staging Manual, 7th Edition. New York, Springer, 2010.

26. Livak KJ and Schmittgen TD: Analysis of relative gene expression data using real-time quantitative PCR and the 2(-Delta Delta C(T)) method. Methods 25: 402-408, 2001
27. Gao J, Aksoy BA, Dogrusoz U, Dresdner G, Gross B, Sumer SO, Sun Y, Jacobsen A, Sinha R, Larsson E, et al: Integrative analysis of complex cancer genomics and clinical profiles using the cBioPortal. Sci Signal 6: pl1, 2013.

28. Hao Y, Triadafilopoulos G, Sahbaie P, Young HS, Omary MB and Lowe AW: Gene expression profiling reveals stromal genes expressed in common between Barrett's esophagus and adenocarcinoma. Gastroenterology 131: 925-933, 2006.

29. Kim SM, Park YY, Park ES, Cho JY, Izzo JG, Zhang D, Kim SB, Lee JH, Bhutani MS, Swisher SG, et al: Prognostic biomarkers for esophageal adenocarcinoma identified by analysis of tumor transcriptome. PLoS One 5: e15074, 2010.

30. Wang S, Zhan M, Yin J, Abraham JM, Mori Y, Sato F, Xu Y, Olaru A, Berki AT, Li H, et al: Transcriptional profiling suggests that Barrett's metaplasia is an early intermediate stage in esophageal adenocarcinogenesis. Oncogene 25: 3346-3356, 2006.

31. Liu YP, Ma L, Wang SJ, Chen YN, Wu GX, Han M and Wang XL: Prognostic value of lymph node metastases and lymph node ratio in esophageal squamous cell carcinoma. Eur J Surg Oncol 36: $155-159,2010$.

32. Yazbeck R, Jaenisch SE and Watson DI: From blood to breath: New horizons for esophageal cancer biomarkers. World $\mathrm{J}$ Gastroenterol 22: 10077-10083, 2016.

33. Duncan SA, Navas MA, Dufort D, Rossant J and Stoffel M: Regulation of a transcription factor network required for differentiation and metabolism. Science 281: 692-695, 1998.

34. Kalkuhl A, Kaestner K, Buchmann A and Schwarz M: Expression of hepatocyte-enriched nuclear transcription factors in mouse liver tumours. Carcinogenesis 17: 609-612, 1996.

35. Chen G, Korfhagen TR, Karp CL, Impey S, Xu Y, Randell SH, Kitzmiller J, Maeda Y, Haitchi HM, Sridharan A, et al: Foxa3 induces goblet cell metaplasia and inhibits innate antiviral immunity. Am J Respir Crit Care Med 189: 301-313, 2014

36. Park SW, Verhaeghe C, Nguyenvu LT, Barbeau R, Eisley CJ, Nakagami Y, Huang X, Woodruff PG, Fahy JV and Erle DJ: Distinct roles of FOXA2 and FOXA3 in allergic airway disease and asthma. Am J Respir Crit Care Med 180: 603-610, 2009.

37. Ma X, Xu L, Gavrilova O and Mueller E: Role of forkhead box protein A3 in age-associated metabolic decline. Proc Natl Acad Sci USA 111: 14289-14294, 2014

38. Shen W, Scearce LM, Brestelli JE, Sund NJ and Kaestner KH: Foxa3 (hepatocyte nuclear factor 3gamma) is required for the regulation of hepatic GLUT2 expression and the maintenance of glucose homeostasis during a prolonged fast. J Biol Chem 276: 42812-42817, 2001.

39. Ang SL, Wierda A, Wong D, Stevens KA, Cascio S, Rossant J and Zaret KS: The formation and maintenance of the definitive endoderm lineage in the mouse: involvement of HNF3/forkhead proteins. Development 119: 1301-1315, 1993.

40. Monaghan AP, Kaestner KH, Grau E and Schütz G: Postimplantation expression patterns indicate a role for the mouse forkhead/HNF-3 alpha, beta and gamma genes in determination of the definitive endoderm, chordamesoderm and neuroectoderm. Development 119: 567-578, 1993.

This work is licensed under a Creative Commons Attribution-NonCommercial-NoDerivatives 4.0 International (CC BY-NC-ND 4.0) License. 